\title{
Influence of temperature inhomogeneity on product profile of reactions occurring within zeolites ${ }^{\mathbb{T}}$
}

\author{
A V ANIL KUMAR ${ }^{1}, \mathrm{~S}$ YASHONATH ${ }^{1,2} *$ and G ANANTHAKRISHNA ${ }^{2,3}$ \\ ${ }^{1}$ Solid State and Structural Chemistry Unit, ${ }^{2}$ Centre for Condensed Matter \\ Theory, and ${ }^{3}$ Materials Research Centre, Indian Institute of Science, \\ Bangalore 560 012, India \\ e-mail: yashonath@sscu.iisc.ernet.in
}

\begin{abstract}
In zeolites, diffusion is often accompanied by a reaction or sorption which in turn can induce temperature inhomogeneities. Monte Carlo simulations of Lennard-Jones atoms in zeolite $\mathrm{NaCaA}$ are reported for the presence of a hot zone presumed to be created by a reaction or chemi- or physi-sorption site. These simulations show that the presence of localized hot regions can alter both kinetic and transport properties such as diffusion. Further, we show that enhancement of diffusion constant is greater for systems with larger barrier height, a surprising result that may be of considerable significance in many chemical and biological processes. We find an unanticipated coupling between reaction and diffusion due to the presence of a hot zone in addition to that which normally exists via concentration. Implications of this coupling for the product profile of a reaction are discussed. We also propose a mechanism by which mobility of ions or diffusion of molecular species within biomembranes may take place.
\end{abstract}

Keywords. Reaction; zeolites; diffusion; temperature inhomogeneity.

\section{Introduction}

Conventional picture of the solid phase is that the constituents pack themselves tightly enough to permit little intervening void spaces. However, there are many solids in nature with voids or pores. These solids may be organic, inorganic or composite materials. Zeolites are archetypal examples of inorganic porous solids with pore sizes comparable to molecular dimensions. ${ }^{1}$ Their study continues to engage the attention of scientists due to their rich and diverse catalytic as well as molecular sieve properties. ${ }^{2}$ Good understanding of these properties requires understanding their diffusion properties. However, diffusion within porous materials or confined geometry is a topic that has been poorly understood $^{3,4}$ despite increased attention in recent times. ${ }^{5,6}$ Life sciences abound with a number of examples which involve diffusion within confined regions, for example, ion diffusion across membranes and approach of a substrate towards an active site of an enzyme. ${ }^{7}$ Hydrocarbon separation and catalysis within zeolites exploited by the petrochemical industries for refining crude oil provide instances of processes in chemistry. ${ }^{8}$ Problems involving fluid flow through porous medium, their influence on transport properties and excitonic transport through porous media are some examples from physical sciences. ${ }^{4}$ The richness of the subject partly arises from the fractal geometry of

\footnotetext{
II Dedicated to Professor C N R Rao on his 70th birthday

*For correspondence
} 
the pores, for instance. Another probable reason is the inherent inhomogeneous nature of the confining geometry. However, while non-uniformity of concentration has been dealt with in great detail in the literature, temperature inhomogeneity has received little attention. In particular, when temperature is inhomogeneous, the very definition of diffusion as being an activated process needs generalisation. Such non-uniformity in temperature arises routinely in biological, chemical and physical systems for a variety of reasons. Here, we discuss issues relating to the possible sources of such hot spots and their influence on transport properties in the context of zeolites.

Chemical heterogeneity of zeolites (arising from substitution of Si by $\mathrm{Al}$ ) and surface curvatures give rise to chemisorption and physisorption sites respectively. The existence of such chemisorption, physisorption and catalytic sites coupled with their poor thermal conductivity could lead to local hot regions. ${ }^{2,9}$ This in turn may be expected to affect both kinetic and diffusion properties. Such a situation can arise in many biosystems as well. For instance, plasma membrane protein-encoding $m$-RNA IST2 has been recently shown to have high asymmetry in concentration between the mother cell and the bud. ${ }^{10}$ One possible way of maintaining such asymmetry against the concentration gradient is through localised hot or cold regions as we demonstrate below. In spite of the importance of such reaction induced hot spots and their possible influence on the diffusion of the species, this problem has not been addressed until recently. ${ }^{11}$

In this paper, we report the study of the effect of inhomogeneous temperature, presumed to be created by a 'reaction', on the equilibration rate and self-diffusion coefficient of guest molecules in zeolites. Here we consider Monte Carlo simulations on simple argon atoms in zeolite A. Our results show that self-diffusion coefficient $D$ is increased substantially due to the presence of a localised hot zone. More significantly, at a conceptual level our analysis shows that local changes in temperature resulting from reactions can induce additional coupling between reaction and diffusion.

\section{Theory}

In a seminal paper ${ }^{12}$ on the relative occupation of the competing local energy minima for a system far from equilibrium, Landauer pointed out the globally determining role played by the non-equilibrium kinetics of the unstable intermediate states even as these are very rarely populated. More specifically, for the case of two local energy minima, i.e., a bistable potential, he showed that the application of a localized heating at a point on the reaction coordinate lying between the lower energy minimum and the potential barrier maximum can raise the relative population of the higher lying energy minimum over that given by the thermal Boltzmann factor $\exp \left(-\Delta E / k_{B} T\right)$. This is the so called 'blowtorch' effect $^{12}$ associated with a non-uniform thermal bath. It generalises the problem of escape of a Brownian particle over a potential barrier under the influence of equilibrium thermal fluctuations, studied originally by Kramers, ${ }^{13-15}$ to the case of non-uniform temperature along the reaction coordinate.

As the blow torch effect is rather counter intuitive, following Landauer, ${ }^{12}$ we illustrate the effect of a hot zone for the case of a bistable potential $U(x)$. Consider the motion of an overdamped particle in this potential (curve ABCD shown as (i) in figure 1) subject to a uniform temperature $T_{0}$ along the coordinate. Then, the probability of finding a particle at $x$ is $P(x) \sim \exp \left(-U(x) / k_{B} T_{0}\right)$. Clearly, the probability at $\mathrm{A}$ is higher than that at $\mathrm{D}$. Now consider raising the temperature of the region $\mathrm{BC}$ to $T_{b}$. Then $P(x) \sim \exp \left(-U(x) / k_{B} T_{b}\right)$ in $\mathrm{BC}$, is much smaller compared to $P(x)$ with $T=T_{0}$. Now consider a situation where the 
population along a unknown potential is given. Then, one can consider the 'potential' $U(x) / k_{B} T=-\log P(x)$. Thus, raising the temperature to $T_{b}$ in $\mathrm{BC}, P(x)$ is much smaller than the population at the ambient temperature $T_{0}$. Then, the corresponding 'potential' is a flatter curve $\mathrm{BC}^{\prime}$ (curve (ii) in figure 1). Since $P(x)$ is unaffected in other regions, the curve outside the region $\mathrm{BC}$ will be the same excepting that the curve $\mathrm{CD}$ would start at $\mathrm{C}^{\prime}$ and end at $\mathrm{D}^{\prime}$ such that $U\left(x_{\mathrm{C}}\right)-U\left(x_{\mathrm{D}}\right)=U\left(x_{\mathrm{C}^{\prime}}\right)-U\left(x_{\mathrm{D}^{\prime}}\right)$. Thus, the point $\mathrm{D}$ is brought down relative to A. Consequently, $P\left(x_{\mathrm{D}}\right)$ is higher than at the lower minimum $x_{\mathrm{A}}$.

A decade later, van Kampen ${ }^{16}$ derived an equation appropriate for the description of diffusion in an inhomogeneous medium where he developed a stochastic treatment for the case of non-uniform temperature. Apart from justifying Landauer's conjecture, he also showed that there could be a net current when the particles are allowed to diffuse back through an alternate route bypassing the hot zone. The problem has also been treated by Büttiker around the same time. ${ }^{17}$ He showed that a net current is possible, even in the absence of an externally applied field, provided both the potential and the state-dependent diffusion constant are periodic with a relative phase difference. Later Landauer ${ }^{18}$ considered this aspect of the problem again in the light of van Kampen's work. Sinha and Moss ${ }^{19}$ have verified Landauer's conjecture by computer simulation. This has also been applied to thermal activation in a superconducting ring with a weak link where transitions produce temperature changes. ${ }^{20}$ Indeed, a whole new field of research has emerged centring on the idea of the possibility of directed motion out of noisy states under diverse athermal driving conditions, generically subsumed under 'thermal ratchets ${ }^{, 21-26}$ and traceable to the original 'blowtorch theorem' of Landauer in the sense that the latter may be viewed as injection of noise. ${ }^{27}$

Most of the investigations on the blowtorch effect study the influence of inhomogeneous temperature profile on the steady-state relative occupations of the energy minima. Recently, Bekele et $a l^{28}$ have shown that the escape rate is enhanced due to the presence of a hot zone. This implies that the rate of surmounting the barrier in zeolite will be increased by the presence of a hot zone and a consequent increase in diffusion constant.

The Smoluchowski equation describing the kinetics of a Brownian particle in an inhomogeneous medium is given by ${ }^{16}$

$$
\frac{\partial P(x, t)}{\partial t}=\frac{\partial}{\partial x}\left[\mu(x)\left[U^{\prime}(x) P(x, t)+\frac{\partial}{\partial x}(T(x) P(x, t))\right],\right.
$$

where the mobility, $\mu$ and temperature, $T$, are in general space-dependent. (We take Boltzmann's constant, $k_{B}=1$.) Here, $P(x, t)$ is the probability density of finding the

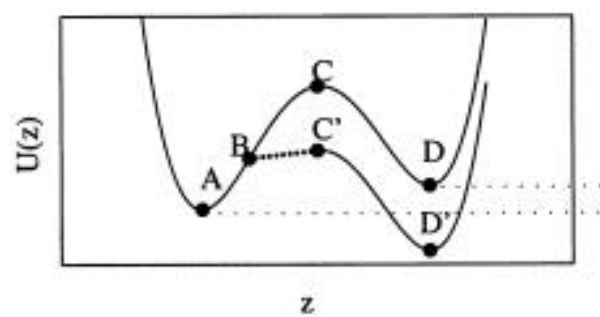

Figure 1. The effect of a hot zone at BC in the potential $A B C D$ is to lower D to D'. 
particle at position $x$ at time $t$ and $U(x)$ describes the potential profile and the prime on $U$ denotes the derivative with respect to $x$.

Some remarks on the use of the Smoluchowski equation, (1), for the case of inhomogeneous medium are in order at this point as the latter has been a subject of some debate that still continues. ${ }^{16,18,27}$ Landauer has argued ${ }^{18,27}$ for a generalisation of the Smoluchowski equation where the second term on the RHS of (1) is to be replaced by $P(x, t)(\partial T(x) / \partial x)+\alpha T(x)(\partial P(x, t) / \partial x)$. The parameter $\alpha$ was shown to depend on the physical conditions to be imposed across the temperature discontinuity for no net current. The parameter $\alpha=0.5$ corresponds to a particle interacting with the thermal bath for which the particle velocity is taken to be proportional to $T^{1 / 2}$ or $P(x) \propto 1 /[T(x)]^{1 / 2}$. On the other hand, $\alpha=1$ corresponds to the case when particles equilibrate via collision (pressure equilibration), i.e., $P(x) \propto 1 / T(x)$. However, a direct derivation based on phase space Smoluchowski equation by van Kampen gave (1) corresponding to $\alpha=1$. This is also supported by the work of Jayannavar and Mahato ${ }^{29}$ based on a microscopic treatment of the thermal bath as a set of harmonic oscillators. In our work, we will continue to use (1) as providing a physically valid description of the problem under consideration.

\section{Monte Carlo simulations}

Zeolites occur in over a hundred or more structural types. Here we have chosen zeolite LTA (also known as A zeolite) for the present investigation. More precisely, the physical system we wish to simulate consists of $\mathrm{NaCaA}$ zeolite with large $(\approx 11.5 \AA$ dia) cages (the supercages) interconnected by shared narrow 8-ring windows $(\approx 4.5 \AA$ dia).The potential energy landscape has a maximum near the 8-ring window and the minimum is located deep within the supercage. A species arriving at a heterogeneous reaction site, assumed to be located between the window and the centre of the cage (see figure 2), releases a heat $q$ creating a local hot zone. Consequently, the molecule surmounts the barrier more easily. Other molecules behind it also cross the barrier with relative ease due to the presence of the hot zone. Here, we mimic the reaction by its principal effect - the presence of a hot zone-by introducing it in between the potential maximum and

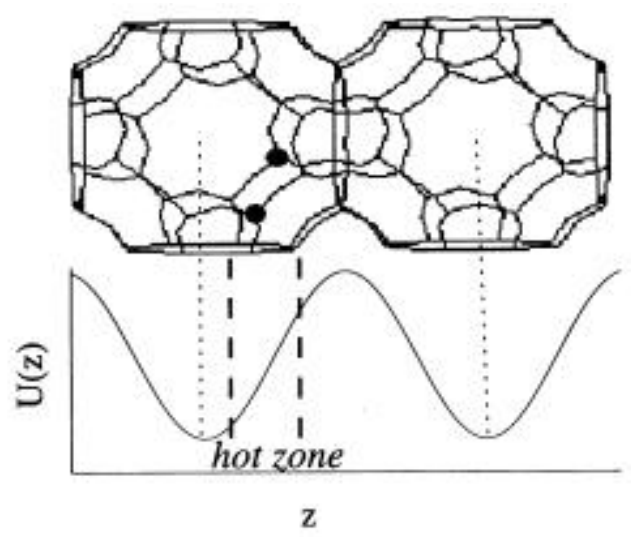

Figure 2. Two cages of zeolite $\mathrm{NaCaA}$. $\bullet$ denote reaction sites. The saddle point is at the window. A schematic one-dimensional potential along the $z$-direction is shown below along with the induced hot zone. 
minimum. Two different sets of simulations A and B are carried out for the purpose of calculation of the escape rate and the diffusion constant D respectively (see below for details).

The choice of the system is Lennard-Jones particles confined to $2 \times 2 \times 1$ unit cells of $\mathrm{NaCaA}$ zeolite. There are $2^{3}=8$ cages in each unit cell. Thus, we have $4 \times 4 \times 2$ cages in the $x, y$ and $z$-directions. $4 \times 4$ cages along $x$-and $y$-directions are chosen to ensure better statistics. Thus, along the $z$-direction the potential is a symmetric double-well potential (see figure 2). Here the diffusion event under consideration is the passage of the diffusant through the 8-ring window, the rate determining step. The diffusion coordinate therefore is the distance of the diffusant from the plane of the window. Simulations are carried out using the Metropolis Monte Carlo algorithm in the canonical ensemble where the total energy is

$$
\Phi=\sum_{g} \sum_{z} \phi_{g z}+\frac{1}{2} \sum_{g} \sum_{g} \phi_{g g}
$$

and $\phi_{a b}=4 \varepsilon_{a b}\left[\left(\sigma_{b b} / r\right)^{12}-\left(\sigma_{b b} / r\right)^{6}\right] ; \quad a, b=g, h$ is the Lennard-Jones (6-12) potential. Thus, the particle diffuses within the zeolite on the potential energy landscape provided by the zeolite. The potential parameters for the guest-guest and guest-host interactions are $\sigma_{g}=2.73 \AA \quad$ and $\varepsilon_{g g}=0.997729 \mathrm{~kJ} / \mathrm{mol} . \quad \sigma_{-\mathrm{O}}=2.5447 \AA, \sigma_{\mathrm{Na}-\mathrm{Na}}=3.369378 \AA$ $\sigma_{\mathrm{Ca}-\mathrm{Ca}}=3.35 \AA, \varepsilon_{\mathrm{O}-\mathrm{O}}=l \times 1.2899134 \mathrm{~kJ} / \mathrm{mol}, \quad \varepsilon_{\mathrm{Na}-\mathrm{Na}}=l \times 0.0392347 \mathrm{~kJ} / \mathrm{mol}$ and $\varepsilon_{\mathrm{Ca}-\mathrm{Ca}}=$ $l \times 9.545046 \mathrm{~kJ} / \mathrm{mol}^{30}$ where $l=2,4$. The cross-terms are obtained from LorentzBerthelot combination rule. Periodic boundary conditions (PBC) are imposed along the $x$ - and $y$-directions only; a repulsive wall with $1 / r^{12}$ potential was used at both ends along $z$-direction with no PBC (set A). No PBC along z-direction enables us to compare our results with earlier results. ${ }^{28}$ Note that in contrast, $\mathrm{PBC}$ along $z$-direction induces a net flow. ${ }^{16}$ The cut-off distance was $12 \AA$. Instead of using the temperature of the hot zone to investigate the influence of the hot zone, it advantageous to another scaled parameter defined by $s=\left(T_{b}-T_{0}\right) / T_{0}$. The barrier height $U_{a}$ is proportional to $l \varepsilon T_{0}$ is kept at $300 \mathrm{~K}$, and $T_{b}$ is varied. $U_{b}$ is varied by varying $l$.

\section{Results and discussion}

To start with, we consider all the 64 guest particles to be uniformly distributed in the four left cages located along the $z$ direction (see figure 1). Then, we obtain the transport properties by calculating the time scales associated with the approach to the steady state. These time scales are obtained by allowing the system to evolve towards the steady state in the presence of the hot zone. Subsequently, when the number of particles in the left and the right cages, denoted by $n_{l}$ and $n_{r}$ respectively, do not change appreciably, the decay rate is obtained by plotting the fraction of particles in the left cages as a function of time. A typical plot of $\ln \left(n_{l} /\left(n_{l}+n_{r}\right)\right)$ vs $t$ is shown in figure 3 . The slope then gives the equilibration rate. The curve shown in figure 3 reaches a plateau at much longer time scales typically around $10^{7} \mathrm{MC}$ steps.

We consider the influence of the hot zone on the rate of approach to the steady state as a function of $s$ when the hot zone of width $w=2 \AA$ is place at a distance $d=1.2775 \AA$ from the window. Instead of using the escape rates, following Bekele et al, we use another parameter called the enhancement factor $f_{b}$ which is the ratio of the escape rates 
from the left cage with and without the hot zone. This quantity is obtained by calculating the escape rates with and without the hot zone and taking their ratio. A plot of $f_{b}$, as a function of $s$, for two different values of $l$ is shown in figure 4 . The tendency to approach saturation is seen for both values of $U_{a}(\propto l \varepsilon)$, even though, it is less pronounced for the higher value $(l=4)$. More importantly, $f_{b}$ is a sensitive function of the barrier height ${ }^{28}$ (figure 4). Thus, the effect will be more pronounced in the guest-host systems with higher energy barrier to diffusion.

The significance of these results becomes apparent on examining a real system such as methane in faujasite. The energy at the physisorption site for methane in $\mathrm{NaY}$ zeolite (with $\mathrm{Si} / \mathrm{Al}=3.0$ ) is $-18 \mathrm{~kJ} / \mathrm{mol}$, the energy difference between a free methane and a physisorbed methane. However, the energy released is significantly lower $(\sim-6 \mathrm{~kJ} / \mathrm{mol})$ when it is already within the zeolite. This can raise the temperature in the vicinity of the site.

We now consider the influence of a hot zone on diffusion. For this, set B simulations were carried out with $\mathrm{PBC}$ in all the three directions and in order to reduce the computational time required, the starting configuration for these were the final configuration of set $\mathrm{A}$ at the same temperature. We have investigated the influence of the parameter $s$, and the barrier height $\left(U_{a} \propto l \varepsilon\right)$. The results are shown in table 1 . The ratio of the diffusion

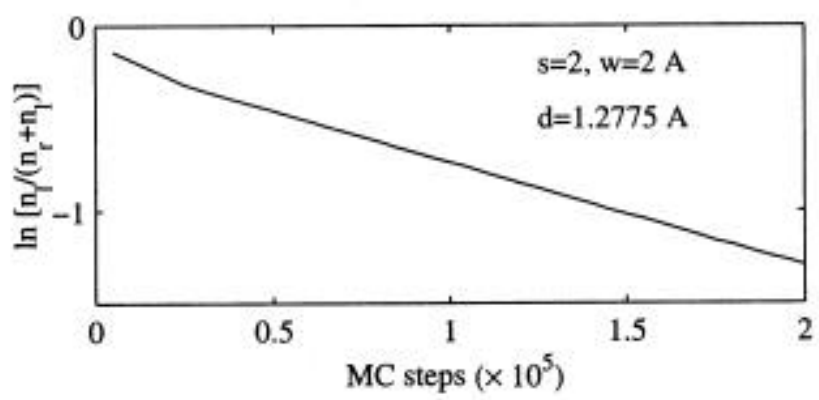

Figure 3. A typical plot of $\ln \left(n_{l} /\left(n_{l}+n_{r}\right)\right)$ vs MC steps obtained from MC runs (set A).

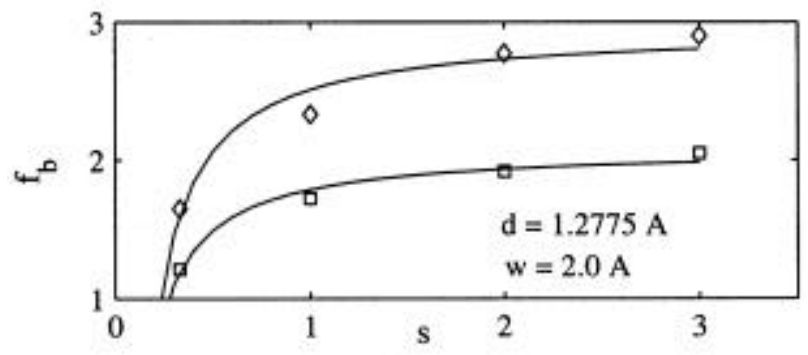

Figure 4. A plot of the enhancement factor, $f_{b}$, as a function of the degree of hotness, $s=\left(T_{b}-T_{0}\right) / T_{0}$, for two different values of well depth, $l \varepsilon(l=2(\square), 4(\diamond))$ from MC runs (set A). 
Table 1. Values of $D_{h} / D_{0}$ for different sets of parameters.

\begin{tabular}{lcccc}
$d=1.2775 \AA$ & $\AA$ & \multicolumn{4}{l}{$\mathrm{A}=2$} & \multicolumn{4}{c}{ (obtained from MC runs (set B)) } \\
\hline$s$ & $l\left(\right.$ in $\left.\varepsilon=l \times \varepsilon_{g z}\right)$ & $n_{l} / n_{l}$ & $D_{h} / D_{0}($ estimated $)$ & $D_{h} / D_{0}(\mathrm{MC})$ \\
\hline 2 & $2 \varepsilon$ & 2.0175 & 1.5088 & 1.4283 \\
3 & $2 \varepsilon$ & 3.5416 & 2.2703 & 2.1971 \\
2 & $4 \varepsilon$ & 2.6132 & 1.8066 & 1.6747 \\
\hline
\end{tabular}

constant with the hot spot $\left(D_{h}\right)$ to that without $\left(D_{0}\right)$ is enhanced in each case. We note that since all other conditions of the simulation are identical in the two situations, $D_{h} / D_{0}$ is independent of the basic length and time scales. Note that the larger the barrier height greater is the enhancement in $D$, a result that has considerable implication. These results are better understood on the basis of simple arguments to estimate $D_{h} / D_{0}$.

We now attempt to explain the enhancement of the diffusion constant in the presence of the hot zone on the basis of Kramers picture. Here, the slowest time scale determining the approach to the steady state is identified with the escape rate. To estimate this, consider a one dimensional symmetric double well potential with PBC. Then the rate equation for the number of particles to the left of the potential maximum $n_{l}$ and that to the right $n_{r}$ is given by

$$
\dot{n}_{l}=-w_{l r} n_{l}+w_{r l} n_{r}
$$

where $w_{l r}$ and $w_{r l}$ are the escape rates from the left to the right well and vice versa respectively. The relevant point here to note is that these are the very time scales that determine the steady state also through detailed balance condition, namely, $\dot{n}_{l}=0$ and $w_{l r} n_{l}=w_{r l} n_{r}$. Now, consider first the situation when there is no hot spot. In this case, $w_{l r}=w_{r l}$ and the mean escape rate from a well is $w_{0}=\left(w_{l r}+w_{r l}\right) / 2$. Then the diffusion constant in one dimension, $D_{0}=a^{2} w_{0} / 2$, where $a$ is the distance between the two minima. Now consider the situation when a hot zone is introduced between the minimum of the left well and the maximum. Then, we have just shown that $w_{l r}$ increase considerably and $w_{r l}$ increases only marginally consistent with the work of Bekele et al. ${ }^{28}$ For further discussion we make the approximation $w_{r l} \approx w_{0}$. Then, the mean escape rate is $w_{h}=\left(w_{l r}+w_{r l}\right) / 2$. Using this with $w_{l r}=w_{r l} n_{r} / n_{l}$, we get $D_{h}=a^{2} w_{h} / 2 \approx D_{0}\left(1+n_{r} / n_{l}\right) / 2$. Since, $n_{r} / n_{l}>1$ in the presence of a hot zone, it is evident that $D$ is enhanced. Note that this refers to a nonequilibrium inhomogeneous situation as $n_{r} / n_{l}$ refers to the steady state which can only be obtained numerically. Table 1 , shows the values of $D_{h} / D_{0}$ obtained from MC simulation of the mean square displacement for three sets of parameter values along with $D_{h} / D_{0}$ estimated from the above expression using the steady state values of $n_{r} / n_{l}$ from MC simulations. (Note $n_{r}$ and $n_{l}$ refers to the total number of particles to the left and right of the potential maxima between the two cages in the $z$-direction.) It is seen that the estimated values of $D_{h} / D_{0}$ are close to that obtained directly from MC. Note that this value is only an upper bound since we have used $w_{r l} \approx w_{0}$ and the asymptotic value of $n_{r} / n_{l}$, which is usually not attained in numerical simulations in spite of long runs. Thus, we find that this relation is a good approximation to ratios of the diffusion constants obtained directly from MC.

To facilitate comparison of these results with real systems, we have estimated the likely increase in temperature when hydrocarbons and other guest species are sorbed 
within zeolites such as $\mathrm{NaX}$. We have listed in table 2 isosteric heat of sorption $\left(\Delta H_{a d s}\right)$ of some linear alkanes, Xe and water within faujasites. We have also listed the mean heat capacities $\left(C_{m}\right)$ of the guest-zeolite systems. ${ }^{1}$ From these data, the maximum increase in $\Delta T$ can be estimated from $\Delta T=T_{b}-T_{0}=\Delta H_{\text {ads }} / C_{m}$ which is in the range 820 to $2028 \mathrm{~K}$ (table 2). Thus, the parameter $s$ varies from 1.7 to 6.7 for which $f_{b}$ can be as large as 3 for $s \sim 6$ and $l=2$. Since $f_{b}$ is determined by the very rate constants that lead to steady state, it also implies that the diffusion constant in the inhomogeneous medium can increase by a factor of two, even for moderate values of $s$ as can be seen from table 1 .

An interplay of reaction and diffusion is known to give rise to complex dynamics which can manifest in different ways. ${ }^{32}$ The product profile in a reaction is controlled by the diffusion rate of the product species formed rather than the reaction rate as is the case with the formation of $p$-xylene in ZSM-5., ${ }^{2,9}$ Due to the low diffusivity of the ortho and meta isomers, they are not observed as products even though they are formed. The coupling between reaction and diffusion in such systems normally is via the concentration of the reactant and product species. ${ }^{33}$ In contrast, in the present situation, the enhancement in $D$ is a direct consequence of inhomogeneous temperature. The present study demonstrates that such a coupling between reaction and diffusion can arise not just due to concentration, but also due to the increase in local temperature, a fact that could not have been anticipated. The results obtained here show how diffusion is increased in the presence of physisorption or chemisorption which are usually exothermic. $D$ may decrease if the reaction is endothermic. Previously, there have been studies on reaction product profiles formed within zeolites. These reactions typically are hydrocarbons undergoing molecular transformations within the cavities of a zeolite. Often, it has been found that the actual reaction products that are measured (essentially outside the zeolite) are $n o t$ the species that are formed within the confined regions. This discrepancy has been explained in terms of the fact that some of the product species are unable to escape from the cavity where they are formed due to either their size or large activation barrier. This leads to building up of the concentration of these species frequently driving the reaction in the opposite direction. It is also possible that other reactions occur when concentrations of selected product species increases. If both concentration and local inhomogeniety in temperature are taken into account while considering diffusion-limited reactions it is

Table 2. Expected rise in temperature and $s$ for typical guests when adsorbed in common zeolites estimated from heat of adsorption, $\Delta H_{a d s}$ and the mean heat capacity, $C_{m}$ data.

\begin{tabular}{lcccccc}
\hline \multicolumn{2}{c}{ System } & & & & & \\
Guest & Zeolite & $\begin{array}{c}\Delta H_{\text {vap }} \\
(\mathrm{kJ} / \mathrm{mol})\end{array}$ & $\begin{array}{c}\Delta H_{\text {ads }}^{a} \\
(\mathrm{~kJ} / \mathrm{mol})\end{array}$ & $\begin{array}{c}C_{m} \\
(\mathrm{~J} / \mathrm{mol} \mathrm{K})\end{array}$ & $\begin{array}{c}T_{b}-T_{0} \\
(\mathrm{~K})\end{array}$ & $s$ \\
\hline$n-\mathrm{C}_{4} \mathrm{H}_{10}$ & $\mathrm{Na}-\mathrm{X}$ & 66 & 174 & 105 & $1689^{\mathrm{b}}$ & $5 \cdot 6$ \\
$n-\mathrm{C}_{7} \mathrm{H}_{16}$ & $\mathrm{Na}-\mathrm{X}$ & 87 & 228 & 176 & $1809^{\mathrm{b}}$ & $6 \cdot 0$ \\
$n-\mathrm{C}_{7} \mathrm{H}_{16}$ & $\mathrm{Na}-\mathrm{X}$ & 87 & 228 & 209 & $1090^{\mathrm{c}}$ & $3 \cdot 3$ \\
$n e o-\mathrm{C}_{5} \mathrm{H}_{12}$ & $\mathrm{Na}-\mathrm{X}$ & 54 & 130 & 129 & $1011^{\mathrm{b}}$ & 3.3 \\
iso- $\mathrm{C}_{8} \mathrm{H}_{18}$ & $\mathrm{Na}-\mathrm{X}$ & 88 & 246 & 185 & $1329^{\mathrm{d}}$ & 4.0 \\
$\mathrm{Xe}$ & $\mathrm{Na}-\mathrm{Y}$ & - & 18 & 22 & $820^{\mathrm{e}}$ & $1 \cdot 7$ \\
$\mathrm{H}_{2} \mathrm{O}$ & $\mathrm{Na}-\mathrm{X}$ & - & 142 & 70 & $2028^{\mathrm{b}}$ & $6 \cdot 7$ \\
\hline
\end{tabular}

${ }^{\mathrm{a} C}$ Calculated from $\Delta H_{\text {vap }}$ and the ratio of $\Delta H_{\text {ads }}$ to $\Delta H_{\text {vap }}$ given in ref. 7 .

${ }^{\mathrm{b}} T_{0}=300 \mathrm{~K},{ }^{\mathrm{c}} T_{0}=333 \mathrm{~K},{ }^{\mathrm{d}} T_{0}=325 \mathrm{~K},{ }^{\mathrm{e}} T_{0}=473 \mathrm{~K}$ 
possible that we might be able to explain the experimentally observed product profiles better or we may see certain unexpected results.

Consider diffusion of ions across biomembranes where energy required is normally obtained from conversion of ATP to ADP. The mechanism of how exactly the energy released by ATP to ADP conversion is utilized in driving diffusion of ions or molecules is not clear. One way the diffusion across biomembranes can be expedited is by the use of blow torch effect of Landauer: if the chemical energy from ATP to ADP conversion can be used to create a hot spot at the appropriate location (e.g., between the energetic minimum and the maximum) then this can facilitate diffusion across the diffusion barrier.

\section{Conclusion}

The present analysis can provide an insight into a well known experimental observation where a warm adsorption front is seen to move rapidly during the adsorption of a gas into an evacuated zeolite. ${ }^{34}$ As the initial molecules arrive at a physisorption site, heat is released which aids the molecules at the front to cross over the energy barrier and propel the gas forward. Zeolites are crystalline solids, and therefore the active sites are located in a periodic manner. Thus, as the front moves further into the zeolite, hot zones are created successively in a periodic manner. This provides a periodic driving force for diffusion which is in addition to that arising from the concentration gradient. This explains the rapid movement of the front. Precise measurements of the speed of the warm front and its temperature can yield valuable experimental data to obtain an insight into the influence of the hot zone on gas/sorbate diffusivities.

Thus, influence of a reaction induced hot zone can affect diffusion in ways which will be important both from fundamental as well as industrial perspective. Though our results are obtained in the context of zeolites, it is evident that these results are of significance to many biological processes where concentration gradients are frequently accompanied by difference in temperature. Additionally, these results have implication to the petrochemical industries, fast ion conducting battery materials, etc. We believe that in the foreseeable future, local inhomogeneities in temperature will be exploited in a number of ways to bring forth novel processes.

\section{Acknowledgements}

We would like to acknowledge Prof C N R Rao, FRS who has been a great source of encouragement for many of us. AVAK and SY would like to thank the Inter-University Consortium, Indore for the award of Project Assistantship and the Department of Science and Technology, New Delhi for financial support.

\section{References}

1. Barrer R M 1978 Zeolites and clay minerals as sorbents and molecular sieves (London: Academic Press)

2. Thomas J M and Thomas W M 1997 Principles and applications of heterogenous catalysis (Weinheim: $\mathrm{VMH}$ )

3. Complete issue of J. Chem. Soc. Faraday Trans. (1991) 87

4. Klafter J and Drake J M (eds) 1989 Molecular dynamics in restricted geometries (New York: Wiley)

5. Bates S P and van Santen R A 1997 Adv. Catal. 421

6. Keil F J, Krishna R and Coppens M-O 2000 Rev. Chem. Eng. 1671 
7. Lehninger A L, Nelson D L and Cox M M 1993 Principles of biochemistry (New York: North Publications)

8. Karger J and Ruthven D M 1992 Diffusion in zeolites and other microporous solids (New York: John Wiley and Sons)

9. Thomas J M 1990 Philos. Trans. R. Soc. London A333 173

10. Brendza R P, Serbes L R, Duffy J B and Saxton W M 2000 Science 2892120

11. Anil Kumar A V, Yashonath S and Ananthakrishna G 2002 Phys. Rev. Lett. 88120601

12. Landauer R 1975 Phys. Rev. A12 636

13. Kramers H A 1940 Physica 7284

14. Hanggi P, Talkner P and Borkovec M 1990 Rev. Mod. Phys. 62251

15. Mel'nikov V I 1990 Phys. Rep. 2091

16. van Kampen N G 1988 IBM J. Res. Dev. 32107

17. Büttiker M 1987 Z. Phys. B68 161

18. Landauer R 1988 J. Stat. Phys. 53233

19. Sinha K and Moss F 1989 J. Stat. Phys. 541411

20. Bol D W and De Bruyn Ouboter R 1988 Physica B154 56

21. Svoboda K, Schmitt C F, Schnapp B J and Block S M 1993 Nature (London) 365721

22. Bartussek R B, Hänggi P and Kissner J G 1994 Europhys. Lett. 28459

23. Astumian R D and Bier M 1994 Phys. Rev. Lett. 721776

24. Doering C B, Horthemke W and Riordan J 1994 Phys. Rev. Lett. 722984

25. Mahato M C and Jayannavar A M 1995 Phys. Lett. A209 21

26. Chialvo D R and Millonas M M 1995 Phys. Lett. A209 26

27. Landauer R 1996 In Fluctuations and order: The new synthesis (ed.) M Millonas (Heidelberg: Springer)

28. Bekele M, Rajesh S, Ananthakrishna G and Kumar N 1999 Phys. Rev. E59 143

29. Jayannavar A M and Mahato M 1995 Pramana - J. Phys. 45369

30. Kiselev A V and Du P Q 1981 J. Chem. Soc., Faraday Trans. II 771

31. Yashonath S, Thomas J M, Nowak A M and Cheetham A K 1988 Nature (London) 331601

32. Lee K-J, McCormick W D, Pearson J E and Swinney H L 1994 Nature (London) 369215

33. Demontis P, Suffritti G B and Tilocca A 1999 J. Chem. Phys. 1115529

34. Basmadjian D, Hu K D and Pan C-Y 1975 Ind. Eng. Chem. Process Dev. 14 328; 14340 